\title{
Eight-and-a-Half Syndrome Presented with Trigeminal Neuralgia
}

\author{
Abdulkadir Koçer, MD, MSc* \\ Department of Neurology, Istanbul Special Bölge Hospital, Istanbul, Turkey
}

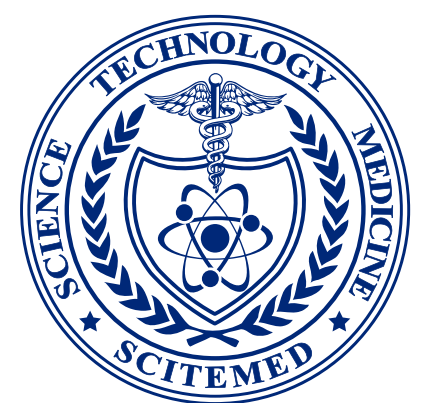

\begin{abstract}
Most isolated syndromes of Eight-and-a-Half Syndrome (EHS) are associated with vascular etiology. Symptomatic trigeminal neuralgia (TN) due to infarction is rare. We report a patient with left-sided facial pain (suddenly developed sharp, electric-shock-like pain attacks lasting for a few seconds). It was followed by one-and-a-half syndrome with facial nerve palsy during the next day. Diffusion-weighted magnetic resonance imaging of his head revealed restricted diffusion in the left inferior pontine tegmentum neighboring the fourth ventricle extending ventrally. Our case is the first report of EHS presented with recurrent attacks of unilateral facial pain, fulfilling criteria for classical TN. It is known that the lesions located in the other brainstem areas, not at the root entry zone, tend to present with atypical facial pain as seen in our patient. Hypertension-related cellular changes and electrophysiological alterations at the pontine level were accepted in etiology.
\end{abstract}

\section{Introduction}

Eight-and-a-half syndrome (EHS) is the combination of one-and-a-half syndromes, which results from injury to either the medial longitudinal fasciculus (MLF) and abducens nerve nucleus or MLF plus the paramedian pontine reticular formation and ipsilateral facial nucleus $[1,2]$. This syndrome is diagnosed clinically, but pontine lesions of a few millimeters of the dorsal pontine tegmentum are characterized radiographically $[1,2]$.

Trigeminal neuralgia (TN) is an uncommon pain disorder, which is defined as a pain condition with short-lasting, most severe attacks in one or more of the peripheral branches, usually due to compression of the retroganglionic trigeminal nerve within the prepontine cistern $[3,4]$. TN due to ischemic lesion of the pons is very rare [3-5]. We present the first reported case of EHS and TN in the same patient.

\section{Case Report}

An elderly patient was admitted to the hospital due to sudden onset of TN, which was followed by the onset of left facial weakness. He had no history of hypertension, diabetes, smoking, and other vascular risk factors. On general physical examination, the only relevant finding was a blood pressure measurement of $145 / 90 \mathrm{mmHg}$. He complained of TN characterized by severe unilateral paroxysmal and 10-60 seconds lasting facial pain attacks, which were stimulated by tactile irritation within the region of the trigeminal nerve on the affected side of the face, not better accounted for by another diagnosis of International Classification of Headache Disorders, remitted and relapsed. The pain was in his left temple presenting typical trigeminal neuralgia over the second and third branches of the nerve and not extending into the left ear. He had gone to a neurologist and he had a history of one gabapentin $600 \mathrm{mg}$ tablet use followed by facial weakness and diplopia within the same day. He did not have similar pain episodes in the past. His neuro-ophthalmological examination showed a combination of left conjugate gaze palsy and left inter-nuclear ophthalmoplegia, suggestive of the horizontal one-anda-half syndrome. Vertical ocular movements from the primary position were normal. Abduction was spared on the right eye. Pupillary reflex was normal on both sides. In addition, he also had left-sided severe facial weakness in a peripheral pattern. He complained of numbness and paresthesia on the left side of the face, but there was not any objective sensory loss. Rest of the neurological examination was normal too.

Diffusion-weighted magnetic resonance imaging (MRI) revealed restricted diffusion in the left inferior pontine tegmentum neighboring the fourth ventricle extending ventrally (Figure 1A and 1B). His initial investigations revealed normal electrolytes, blood profile, and liver functions. Erythrocyte sedimentation rate and treponemal antibody test did not show any abnormality either. Only serum low-density lipoprotein cholesterol level was high $(180 \mathrm{mg} / \mathrm{dL})$. The detailed cardiac exam was normal. He confirmed that the symptoms related to EHS started after using gabapentin and did not want to use any drug for the treatment of neuralgia. Spontaneous recovery of TN was observed within 3 days after drug treatment of mild to moderate level hypertension. Cranial MRI and carotid and vertebral-basilar system computed tomography angiography exams were normal. It was accepted as a small vessel infarction and the treatment with anti-aggregating (acetylsalicyclic acid $300 \mathrm{mg}$ ), anti-hypertensive, and anti-lipidemic agents was started. Gaze palsy and facial weakness persisted in his final neurological examination. Only adduction delay in the left eye was slightly improved. He was subsequently discharged to an inpatient rehabilitation center.

\section{Discussion}

EHS is a combination of ipsilateral $6^{\text {th }}$ and $7^{\text {th }}$ lower motor neuron palsy, internuclear ophthalmoplegia, and ipsilateral gaze palsy $[2,6,7]$. The syndrome can also be described as one-and-a-half syndrome accompanied by $7^{\text {th }}$ lower motor neuron palsy. Most isolated syndromes are associated with vascular etiology, especially pontine lacunar infarction due to occlusion of small penetrating arteries [8]. The other causes are demyelination, vasculitis, tumors, and infectious and basilar artery aneurysm $[8,9]$. Similar to previous reports, we found that a small pontine lesion resulted in EHS (Figure 1A and 1B), but the patient had left-sided TN, which was the first case in the literature.

It is well known that the nuclei of the $5^{\text {th }}$ nerve are located above the $6^{\text {th }}$ and $7^{\text {th }}$ nerve nuclei and laterally to the MLF. Lesions sited at the midpons can affect the main sensory and motor nuclei of the $5^{\text {th }}$ nerve turning up with ipsilateral masticatory muscle paralysis and sensory deficit at ipsilateral face $[3,4]$. In our patient, he had a complaint of atypical sensation and paresthesia, but a sensory deficit in a peripheral trigeminal distribution or in an onion shell-like manner, as known by the involvement of the trigeminal brain stem nuclei, was not found in the examination. In the current case, the trigeminal symptoms were protopathic and had a somatotopic structure. We thought that these trigeminal symptoms possibly emerged from the involvement of the rostral and intermediate portion of the descending trigeminal nucleus, or from some involvement of the root entry zone where somatotopy was known. 


\section{Summary and Conclusion}

Although TN can affect any division alone or in combination, most cases involve both the maxillary and mandibular divisions simultaneously, as seen in our case. TN is most commonly caused by vascular compression of the trigeminal nerve root entry zone. More central lesions usually cause constant burning pain, which rather represents painful trigeminal neuropathy with sensory deficits. Secondary trigeminal neuralgia due to the ischemic lesion of the pons is very rare. The mechanism of TN due to lesions is still a matter of debate. It is believed that TN is due to an irritation at the trigeminal nucleus [4]. Another theory is that the infarction related electrophysiological alterations in the trigeminal system lead to ephaptic transmission, spontaneous firing, and mechanosensitivity in the patients with pontine infarction [7]. The lesions located at the root entry zone present with typical TN, whereas those with lesions located in other brainstem areas tend to present with atypical facial pain [3-5].

In our case, there was no evidence of trigeminal injury and the MRI restricted diffusion did not really overlap with an expected location of the trigeminal nucleus or tract. While secondary TN was typically associated with lesions at the trigeminal dorsal root entry zone, our case was interesting in that the infarct was in the dorsal pons and we accepted that $\mathrm{TN}$ and allodynia on the face were because of infarction and hypertension-related cellular changes and electrophysiological alterations at the pontine level $[3,4]$. So far, as the trigeminal protopathic symptoms were involved, they were possibly the manifestation of ischemic symptoms in the territory of a pontine paramedian penetrating artery. We diagnosed the condition recurrent attacks of unilateral facial pain fulfilling criteria for classical TN and our case was an interesting combination of EHS and TN [10].
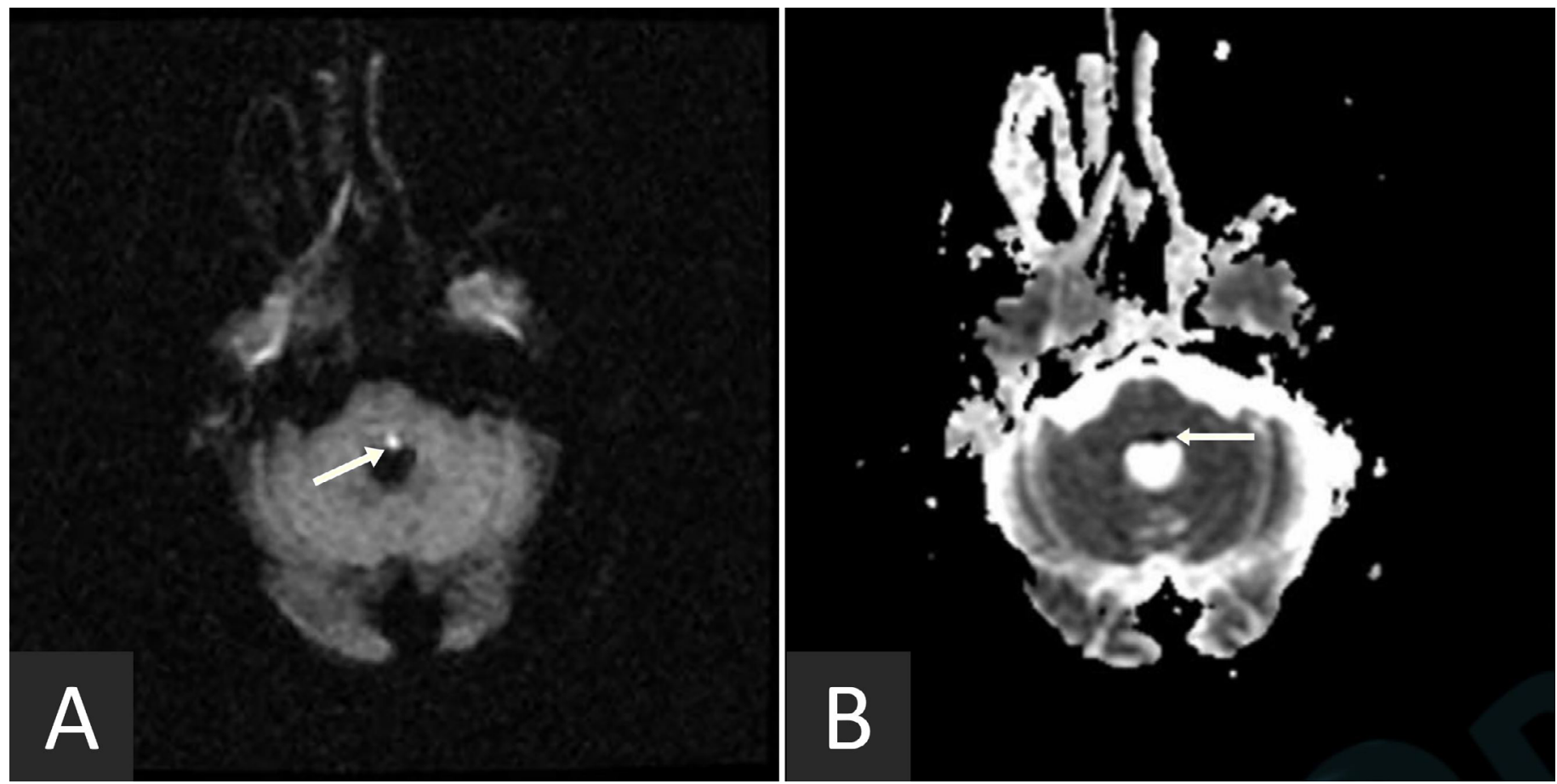

Figure 1. Diffusion-weighted magnetic resonance imaging revealed hyperintensity in the left inferior pontine tegmentum neighboring the fourth ventricle extending ventrally (arrow in panel A). Corresponding low signal was observed on the attenuated diffusion coefficient map (arrow in panel B).

\section{Article Inormation}

*Correspondence: Abdulkadir Koçer, MD, MSc

Namik Kemal Mah, Kirişhane Cad, İstanbul Sitesi, 20/24, Istanbul, Turkey. Email: abdulkadirkocer@yahoo.com

Received: Jul. 16, 2018; Accepted: Aug. 09, 2018; Published: Oct. 23, 2018

\section{DOI: 10.24983/scitemed.acri.2018.00088}

Copyright (c) 2018 The Author. This is an open-access article distributed under the terms of the Creative Commons Attribution 4.0 International License (CC-BY).

\section{Funding: None}

\section{Conflict of Interest: None}

Informed Consent: An informed consent was obtained from the patient.

\section{Keywords}

Eight-and-a-half syndrome; facial palsy; hypertension; infarction; neuralgia; one-and-a-half syndrome, trigeminal neuralgia.

\section{References}

1. Sarwal A, Garewel M, Sahota S, Sivaraman M. Eight-and-a-half syndrome. J Neuroimaging 2009;19(3):288-290.

2. Eggenberger E. Eight-and-a-half syndrome: one-and-a-half syndrome plus cranial nerve VII palsy. J Neuroophthalmol 1998;18(2):114-116.

3. Katsuno M, Teramoto A. Secondary trigeminal neuropathy and neuralgia resulting from pontine infarction. J Stroke Cerebrovasc Dis 2010;19(3):251-252.

4. Peker S, Akansel G, Sun I, Pamir NM. Trigeminal neuralgia due to pontine infarction. Headache 2004;44(10):1043-1045.

5. Arrese I, Lagares A, Alday R, Ramos A, Rivas JJ, Lobato RD. Typical trigeminal neuralgia associated with brainstem white matter lesions on MRI in patients without criteria of multiple sclerosis. Acta Neurochir (Wien) 2008;150(11):1157-1161.

6. Kakar P,Brown Z, Banerjee S. Eight-and-a-half syndrome :an unusual presentation brainstem infarction. QJM 2013;106(3):273276.

7. Marquart C, Strauss C, Alfieri A. Eight-and-a-half syndrome combined with an ipsilateral vertical gaze palsy: a pathophysiological explanation. Clin Neurol Neurosurg 2013;115(6):767-769. 


\section{CASE REPORT}

8. Rosini F, Pretegiani E, Guideri F, Cerase A, Rufa A. Eight and a half syndrome with hemiparesis and hemihypesthesia: the nine syndrome? J Stroke Cerebrovasc Dis 2013;22(8):e637-e638.

9. van Toorn R, Schoeman JF, Donald PR. Brainstem tuberculoma presenting as eight-and-a-half syndrome. Eur J Paediatr Neurol
2006;10(1):41-44.

10. Headache Classification Committee of the International Headache Society (IHS). The International Classification of Headache Disorders, $3^{\text {rd }}$ edition (beta version). Cephalalgia 2013;33(9):629808. 